\title{
Veredas: uma importante fitofisionomia do Cerrado para briófitas
}

\author{
Veredas: an important Cerrado phytophysiognomy for bryophytes \\ Veredas: una importante fitofisiognomía del Cerrado para briófitas
}

\section{Resumo}

O presente estudo teve como objetivo conhecer as briófitas que ocorrem em Veredas da região sul de Goiás e de Minas Gerais, Brasil. As coletas foram realizadas de fevereiro a dezembro de 2014, em quatro áreas de Veredas: duas em Quirinópolis, uma em Gouvelândia (Goiás) e uma em Monte Alegre de Minas (Minas Gerais). Foram encontradas 21 famílias, 27 gêneros e 36 espécies (15 hepáticas, 20 musgos e 1 antócero). Quatro espécies são novos registros para o estado de Goiás e dois novos registros para o estado de Minas Gerais.

Palavras-chave: Antóceros; Terras alagadas; Hepáticas; Musgos; Cerrado.

\begin{abstract}
The present study aimed to know the bryophytes occurring in Veredas of the southern region of Goiás and Minas Gerais, Brazil. The collection was performed from February to December 2014, in four areas of Veredas: two in Quirinópolis, one in Gouvelândia (Goiás) and one in Monte Alegre de Minas (Minas Gerais). Twenty one families, 27 genera and 36 species (15 hepatic, 20 mosses and 1 hornworts) were found. Four species are new records for the state of Goiás, and two new records for the state of Minas Gerais.
\end{abstract}

Keywords: Hornworts; Wetlands; Liverworts; Mosses; Brazilian Savanna.

\section{Resumen}

Este estudio tuvo como objetivo conocer las briofitas que ocurren en Veredas en la región sur de Goiás y Minas Gerais, Brasil. Las muestras se recolectaron de febrero a diciembre de 2014, en cuatro áreas de Veredas: dos en Quirinópolis, una en Gouvelândia (Goiás) y una en Monte Alegre de Minas (Minas Gerais). Encontramos 21 familias, 27 géneros y 36 especies (15 hepáticas, 20 musgos y 1 antoceros). Cuatro especies son nuevos registros para el estado de Goiás y dos nuevos registros para el estado de Minas Gerais.

Palabras clave: Antoceros; Humedales; Hepáticas; Musgos; Cerrado.

\section{Introdução}

O ecossistema do Cerrado abrange $21 \%$ do território brasileiro, constituindo a maior área de savana do mundo (Semarh 2005). Está localizado numa posição geográfica central no Brasil e devido à sua altitude e ao grande número de nascentes fornece a este ecossistema o berço de grandes rios brasileiros (Bastos \& Ferreira 2010). A vegetação inclui florestas secas, savanas, florestas ripárias e campos (Ribeiro \& Walter 2008).

Dentre as formações de savana, destaca-se a vereda, que é uma formação importante devido à proteção das nascentes (Silva \& Maillard 2011). É individualizada devido à sua hidromorfologia, com solos argilosos, geralmente orgânicos, como 
pântanos sazonais e / ou permanentes. Nesse ambiente é comum a ocorrência de Mauritia flexuosa L.f. (buriti), espécie esparsamente entre extensas áreas do estrato herbáceo-gramíneo (Ribeiro \& Walter 2008), e tornando-se mais abundante e adensada próximo ao canal de drenagem. O componente da flora é caracterizado por espécies adaptadas à grande umidade do solo conforme descrito por Resende et al. (2013), entre elas as de briófitas.

As briófitas são o segundo maior grupo de plantas terrestres, sendo consideradas pioneiras na transição do ambiente aquático para o terrestre (Vanderpoorten \& Goffinet 2009). Essas plantas também são de grande interesse ecológico, constituindo-se em indicadores ecológicos valiosos, visto que são muito sensíveis às mudanças nas condições ambientais e, portanto, utilizadas principalmente como indicadores de poluição (Shepherd 2003).

Em todo o mundo, 17.900 espécies de briófitas são estimadas (Gradstein et al. 2001). No Brasil, já são conhecidas 1600 espécies (Flora do Brasil 2020). Atualmente, 490 espécies estão listadas para o bioma Cerrado, das quais 317 são listadas para o estado de Goiás e 369 para o estado de Minas Gerais (Flora do Brasil 2020).

A brioflora goiana ainda é pouco conhecida, pois novas ocorrências são sempre encontradas durante os inventários, como no Parque Estadual da Serra dos Pirineus, 68 espécies com 21 novos registrados (Sousa et al. 2010) e, no estudo realizado em Quirinópolis, 34 espécies com três novos registros (Aquino et al. 2015). Em outro levantamento no Parque Estadual de Caldas Novas, foram registradas 36 espécies, incluindo 25 gêneros e 16 famílias (Carvalho et al. 2014).

Especificamente para o ambiente de veredas em Goiás, apenas sete briófitas foram registradas no município de Bela Vista de Goiás no inventário de todas as plantas, mas ainda assim, o musgo Leucoloma serrulatum Brid. foi um novo recorde para o estado (Resende et al. 2013).

Para a região de Cerrado do estado de Minas Gerais, embora haja mais esforços de pesquisa com briófitas, ainda há necessidade de mais estudos, uma vez que existe uma grande faixa de transição entre Cerrado e Mata Atlântica. Dentre os levantamentos que já foram realizados em áreas que incluem Cerrado são conhecidos Yano e Carvalho (1995), que encontraram 46 espécies na Serra da Piedade; Yano e Peralta (2009), com 42 espécies de musgos e hepáticas em Grão- Montanhas Mogol; Yano e Peralta (2011a) com 114 espécies na Serra de São José, na cidade de Tiradentes; no Parque Nacional da Serra do Cipó, foram registradas 237 espécies (Yano \& Peralta 2011b), o mesmo local, no estudo de (Sousa \& Câmara 2015), foram registradas 12 novas ocorrências para o estado; Luizi-Ponzo et al. (2013) com 209 espécies encontradas no Parque Estadual do Ibitipoca; e Carmo e Peralta (2016) encontraram 289 espécies para a Serra da Canastra.

A brioflora desempenha um papel fundamental nas veredas, constituindo micro-habitats para pequenos invertebrados, sendo valiosos indicadores do meio ambiente e armazenando grande quantidade de carbono, porém pouca informação sobre a composição e ecologia desses vegetais é conhecida. Os levantamentos de briófitas nessas áreas são extremamente importantes para enriquecer o conhecimento e ampliar a distribuição geográfica das espécies. Apesar da importância das veredas para a manutenção dos recursos hídricos e da biodiversidade no Cerrado, os estudos sobre essa fitofisionomia são escassos e revelam a necessidade de aumentar o número de obras nesses ambientes.

Devido ao pequeno conhecimento sobre as briófitas em ambientes de veredas, este estudo foi realizado em quatro localidades de vereda na região sul de Goiás e Minas Gerais, Brasil, visando conhecer a composição e a estrutura da comunidade.

\section{Metodologia}

Foram estudadas quatro áreas de Veredas, duas no município de Quirinópolis (V1 e V2), uma no município de Gouvelândia (V3), no estado de Goiás, e uma no município de Monte Alegre de Minas (V4), no estado de Minas Gerais. A descrição física, área e coordenadas estão na Tabela 1 e Figura 1. As quatro veredas são circundadas por pasto e apresentam perturbações devido ao pastejo e pisoteio do gado e, em algumas, também foram observadas intervenção através de drenagem. 
Tabela 1. Características das áreas das quatro veredas V1 - Boa Sorte, V2 - Retiro (Quirinópolis), V3 - São José (Gouvelândia) e V4 (Monte Alegre de Minas) quanto à localização, tamanho da área e uso antrópico.

\begin{tabular}{|l|l|l|l|l|l|l|}
\hline Vereda & Sigla & $\begin{array}{l}\text { Município / } \\
\text { Estado }\end{array}$ & $\begin{array}{l}\text { Área } \\
\text { (ha) }\end{array}$ & Altitude & Coordenadas & Características da área \\
\hline $\begin{array}{l}\text { Fazenda Boa } \\
\text { Sorte/Serra da } \\
\text { Confusão do Rio } \\
\text { Preto }\end{array}$ & V1 & Quirinópolis, GO & 18,4 & $\begin{array}{l}750 \text { a } 765 \\
\mathrm{~m}\end{array}$ & $\begin{array}{l}18^{\circ} 20^{\prime} 01,18^{\prime \prime} \text { 'S- } \\
50^{\circ} 30^{\prime} 19,51^{\prime \prime O}\end{array}$ & $\begin{array}{l}\text { Vegetação com acesso de gado na borda e no meio, com } \\
\text { início da formação de mata ciliar em alguns trechos na } \\
\text { parte inferior. Localizada a 15 km da zona urbana de } \\
\text { Quirinópolis. }\end{array}$ \\
\hline Fazenda Retiro & V2 & Quirinópolis, GO & 47,2 & $\begin{array}{l}556 \text { a } 565 \\
\mathrm{~m}\end{array}$ & $\begin{array}{l}18^{\circ} 30^{\prime 2} 28,48^{\prime \prime S}- \\
50^{\circ} 52^{\prime 2} 29,38^{\prime \prime O}\end{array}$ & $\begin{array}{l}\text { Vegetação com início de formação de mata de galeria em } \\
\text { alguns trechos ao fundo, com acesso de gado na borda e } \\
\text { meio. Localizada a 47 km da área urbana, essa vereda é } \\
\text { dividida, no sentido leste-oeste, pela rodovia GO-206. }\end{array}$ \\
\hline Fazenda São José & V3 & $\begin{array}{l}\text { Gouvelândia, } \\
\text { GO }\end{array}$ & 28,7 & $\begin{array}{l}429 \text { a } 431 \\
\mathrm{~m}\end{array}$ & $\begin{array}{l}18^{\circ} 25^{\prime} 37,24 " S- \\
50^{\circ} 07^{\prime} 58,59 " O\end{array}$ & $\begin{array}{l}\text { Vegetação com mata ciliar ao fundo; com acesso para gado } \\
\text { na margem e três ralos na margem direita. Localizada a 1,5 } \\
\text { km da área urbana de Gouvelândia, no município de } \\
\text { Cabeceira do Córrego Carvalho. }\end{array}$ \\
\hline $\begin{array}{l}\text { Fazenda em Monte } \\
\text { Alegre de Minas }\end{array}$ & V4 & $\begin{array}{l}\text { Monte Alegre de } \\
\text { Minas, MG }\end{array}$ & 25,4 & $\begin{array}{l}769 \text { a } \\
780 \mathrm{~m}\end{array}$ & $\begin{array}{l}18^{\circ} 49^{\prime} 38,35^{\prime \prime S}- \\
48^{\circ} 49^{\prime} 03,33^{\prime \prime O}\end{array}$ & $\begin{array}{l}\text { Vegetação antropizada, mas com melhor estado de } \\
\text { conservação do que outras áreas, com mata de galeria em } \\
\text { alguns trechos. Distante 9km da zona urbana de Monte } \\
\text { Alegre de Minas. }\end{array}$ \\
\hline
\end{tabular}

Fonte: Autores.

Figura 1. Mapa de localização das quatro áreas das veredas estudadas. V1: Fazenda Boa Sorte, Quirinópolis - GO. V2: Fazenda Retiro, Quirinópolis - GO. V3: Fazenda São José, Gouvelândia - GO. V4: Fazenda em Monte Alegre, MG.

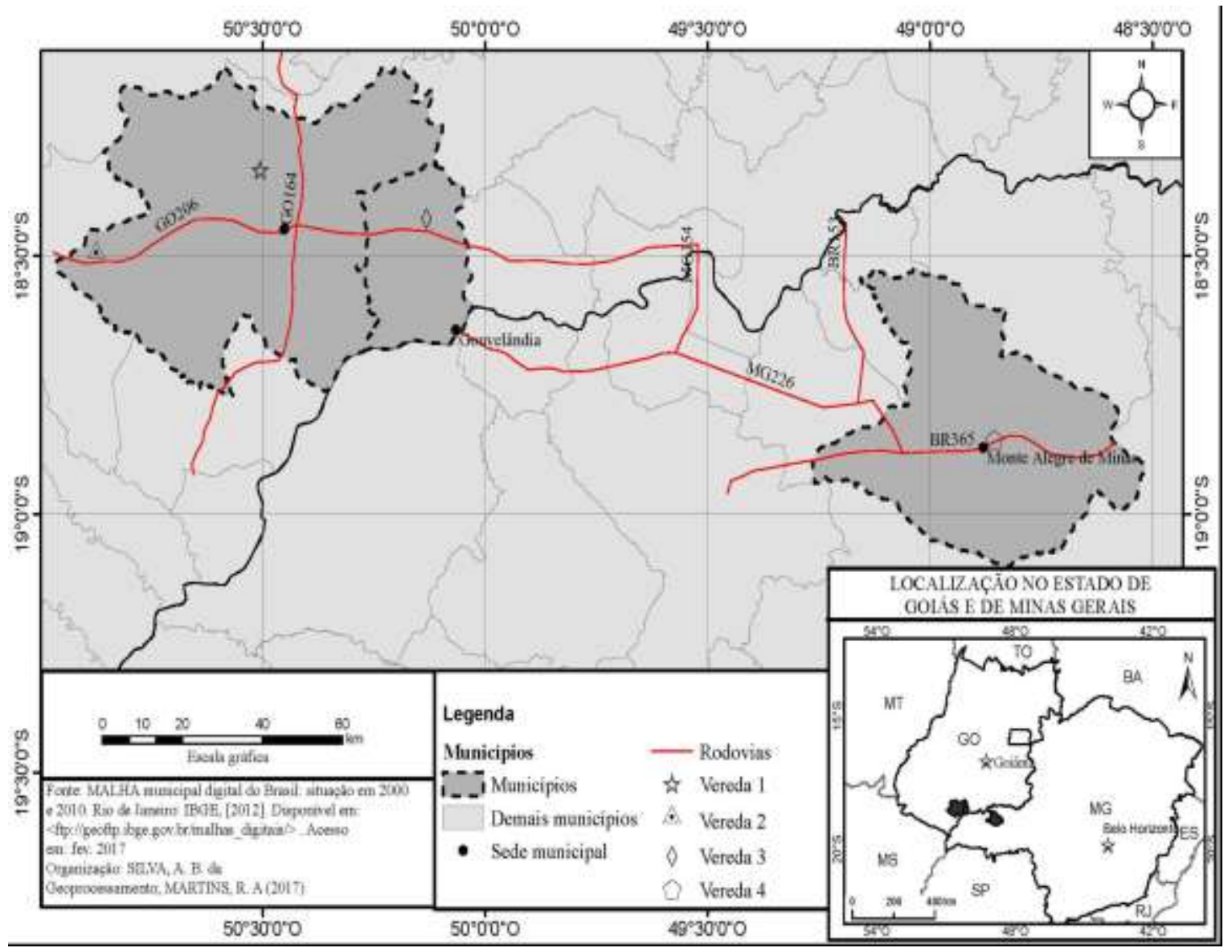

Fonte: Autores. 
As coletas das plantas foram realizadas de fevereiro a dezembro de 2014 utilizando a metodologia descrita em Frahm (2003) e o método de caminhada (Filgueiras et al. 1994), com 24 viagens a campo, sendo seis viagens em cada Vereda com duração de 5 horas cada. A curva de rarefação levou em consideração o número de espécies encontradas pelo número de áreas amostradas e do erro padrão estimado por meio do programa EstimateS 9.10 (Colwell 2013).

As amostras foram herborizadas e incorporadas ao acervo do Herbário José Ângelo Rizzo (JAR), da Universidade Estadual de Goiás, Câmpus Sudoeste, Sede Quirinópolis,. A identificação das amostras baseou-se nos trabalhos de Yano (1975); Vital (1980); Reese (1993); Sharp et al. (1994); Gradstein (1994); Buck e Goffinet (2000); Crandall-Stotler e Stotler (2000); Gradstein et al. (2001); Buck (2003); Gradstein e Costa (2003); Stotler e Crandall-Stotler (2005) e Pursell (2007) e comparados com as amostras já identificadas depositadas em herbário. O sistema de classificação adotado foi baseado em Crandall-Stotler et al. (2009) para Bryophyta; em Goffinet et al. (2009) para Marchantiophyta e Stotler e Crandall-Stotler (2005) para Anthocerophyta. A distribuição geográfica nos estados brasileiros segue a lista de espécies da Flora do Brasil (Flora do Brasil 2020).

A frequência absoluta das espécies foi determinada com base no número de ocorrências total, e classificadas em cinco classes assim definidas: 1-5 $=\mathrm{R}$ - raras; 6-10 $=\mathrm{I}$ - incomum; 11-20 = A - assíduo; 21-30 $=\mathrm{F}$ - frequente e $>30=\mathrm{M}-$ muito frequente (Silva \& Pôrto 2007).

As espécies foram classificadas, de acordo com o local de coleta, em: corticícolas (co - em tronco vivo de árvore); epíxilas (ep - em tronco morto ou em decomposição) e terrestres (te - em solo úmido) (Robbins 1952). E classificadas como generalistas para espécies com um amplo nicho ecológico (colonizando vários tipos de substrato) e especialistas para aquelas com um nicho ecológico restrito (colonizando apenas um substrato) (Ricklefs 2010).

A similaridade florística entre as veredas estudadas foi realizada com base em zonas (diferenciadas de acordo com a inclinação e drenagem do solo: borda - local de solo mais seco; meio - com o solo moderadamente úmido; fundo - local saturado com água e próximo ao canal de drenagem) pelo índice de similaridade qualitativa de Jaccard (Müller-Dombois \& Ellenberg 1974, Oliveira et al. 2009).

Para comparar o número de espécies comuns entre as áreas, o Diagrama de Venn (Venn 1984) foi usado. A análise de agrupamento foi realizada usando o índice de similaridade de Jaccard e usando o link médio de grupo (UPGMA) através do programa PAST (Hammer et al. 2001), sendo o nível de corte para o dendrograma de similaridade de 50\%.

\section{Resultados e Discussão}

Nas quatro áreas de veredas, foram encontradas 36 espécies registradas em 891 ocorrências (Tabela 2). De acordo com a curva de rarefação (Figura 2), provavelmente existam mais espécies, e acreditamos que a riqueza da comunidade não foi alcançada devido ao número de veredas estudado. 


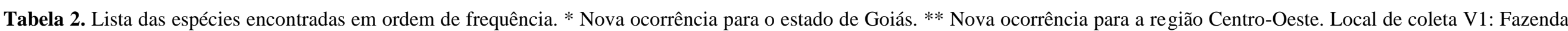

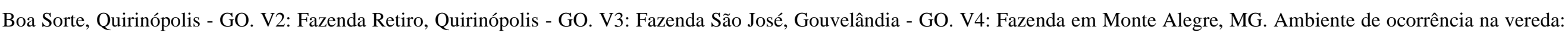

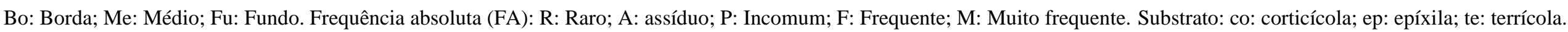

\begin{tabular}{|c|c|c|c|c|c|c|c|c|c|c|c|c|c|c|c|c|c|c|c|c|c|}
\hline & V1 & & & V2 & & & V3 & & & V4 & & & & Dist & ibuiça & & & trate & & & \\
\hline Espécies & Bo & Me & Fu & Bo & Me & Fu & Bo & Me & Fu & Bo & Me & Fu & FA & Bo & Me & $\mathbf{F u}$ & co & ep & te & Voucher & Dist. Geo. \\
\hline Isopterygium tenerifolium Mitt. & 2 & 98 & & & 24 & & 4 & 22 & & 13 & 40 & 15 & $\begin{array}{l}\text { M } \\
(218)\end{array}$ & 19 & 184 & 15 & 1 & 1 & 1 & $\begin{array}{l}\text { Santos } \\
102\end{array}$ & $\begin{array}{l}\text { AM, PA, RO, BA, CE, DF, GO, MT, } \\
\text { MG, RJ, SP, RR, RS e SC. }\end{array}$ \\
\hline $\begin{array}{l}\text { Riccardia chamedryfolia (With.) } \\
\text { Grolle }\end{array}$ & 38 & & & 4 & 50 & & & 1 & & 3 & 65 & & $\begin{array}{l}M \\
(161)\end{array}$ & 45 & 116 & 0 & 1 & 1 & 1 & $\begin{array}{l}\text { Santos } \\
128\end{array}$ & DF, GO, MT, MG, ES, RJ, SP, PR e RS. \\
\hline Lejeunea glaucescens Gottsche** & 2 & 13 & & & 35 & & 3 & 9 & 3 & & 38 & & $\begin{array}{l}\text { M } \\
(103)\end{array}$ & 5 & 95 & 3 & 1 & 1 & 1 & $\begin{array}{l}\text { Resende } \\
2496\end{array}$ & $\begin{array}{l}\text { AC, PA, RO, AL, BA, PE, MS, MT, ES, } \\
\text { RJ, SP e PR. }\end{array}$ \\
\hline $\begin{array}{l}\text { Ectropothecium leptochaeton } \\
\text { (Schwägr.) W.R.Buck }\end{array}$ & & 11 & & & & & & 39 & & & 21 & & M (71) & 0 & 71 & 0 & 1 & 1 & 1 & $\begin{array}{l}\text { Santos } \\
28\end{array}$ & $\begin{array}{l}\text { AM, PA, BA, MS, MT, ES, MG, RJ, PR } \\
\text { e SC. }\end{array}$ \\
\hline Sphagnum subsecundum Nees & & 29 & & 6 & 30 & & & & & & & & $\mathrm{M}(65)$ & 6 & 59 & 0 & 1 & 0 & 0 & $\begin{array}{l}\text { Resende } \\
1863\end{array}$ & $\begin{array}{l}\text { RO, TO, BA, DF, GO, MT, ES, MG, RJ, } \\
\text { SP, PR e SC. }\end{array}$ \\
\hline Isopterygium affusum Mitt. & 10 & 22 & & 5 & 12 & 4 & & 2 & & & & & M (55) & 15 & 36 & 4 & 1 & 0 & 0 & $\begin{array}{l}\text { Santos } \\
43\end{array}$ & AM, PA, GO, MT, MG, RJ, SP e SC. \\
\hline $\begin{array}{l}\text { Philonotis sphaerocarpa (Hedw.) } \\
\text { Brid. }\end{array}$ & & 6 & 1 & & & & & 46 & & & & & M (53) & 0 & 52 & 1 & 1 & 1 & 1 & Santos 3 & AM, BA, CE, MT, RJ, SP e SC. \\
\hline Symphyogyna brasiliensis Nees & & 6 & & & & 6 & & & 6 & & & 12 & $\mathrm{~F}(30)$ & 0 & 6 & 24 & 1 & 1 & 1 & $\begin{array}{l}\text { Santos } \\
305\end{array}$ & $\begin{array}{l}\text { RO, RR, CE, DF, GO, MT, ES, MG, RJ, } \\
\text { SP, PR, RS e SC. }\end{array}$ \\
\hline
\end{tabular}




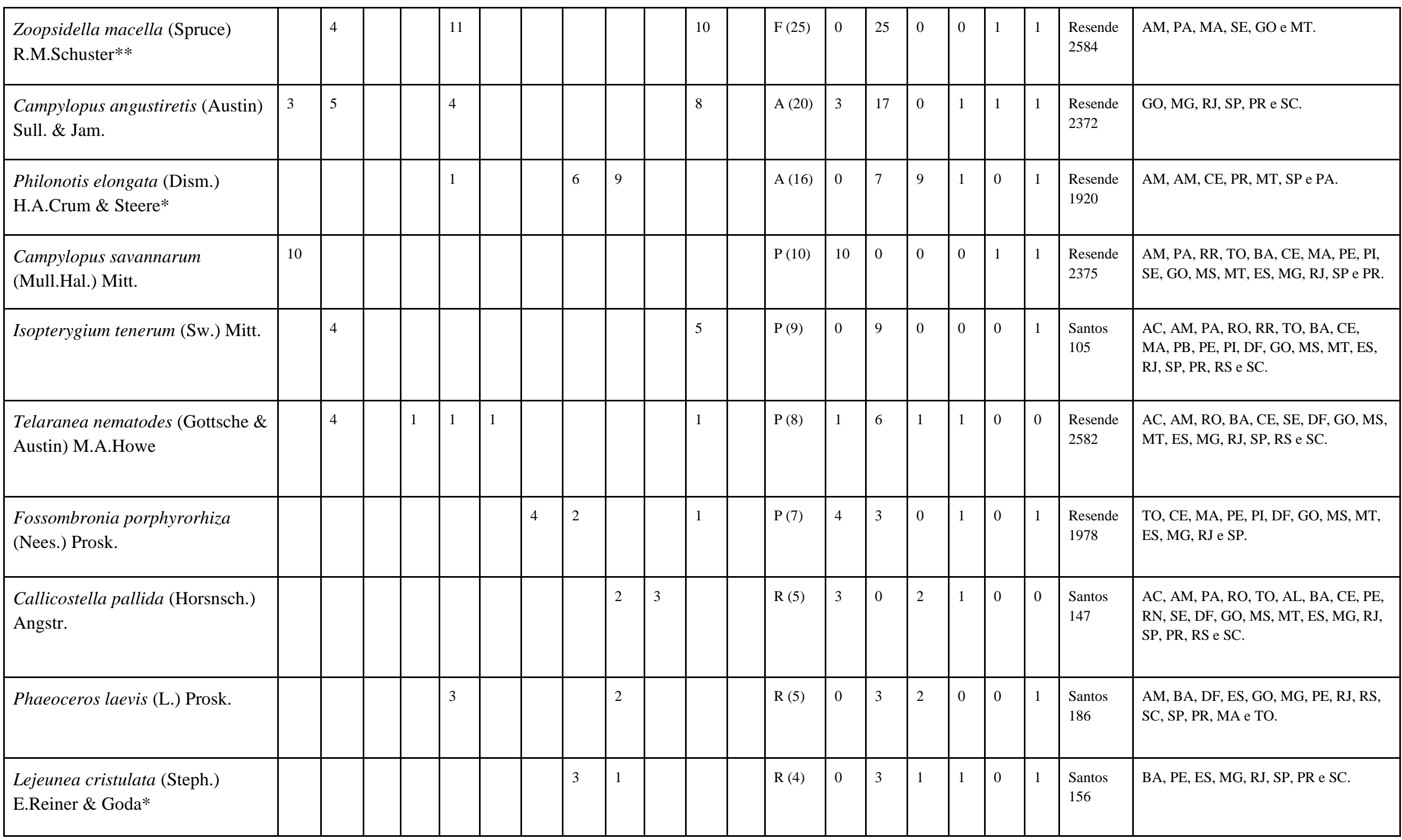


Research, Society and Development, v. 10, n. 11, e268101119474, 2021

(CC BY 4.0) | ISSN 2525-3409 | DOI: http://dx.doi.org/10.33448/rsd-v10i11.19474

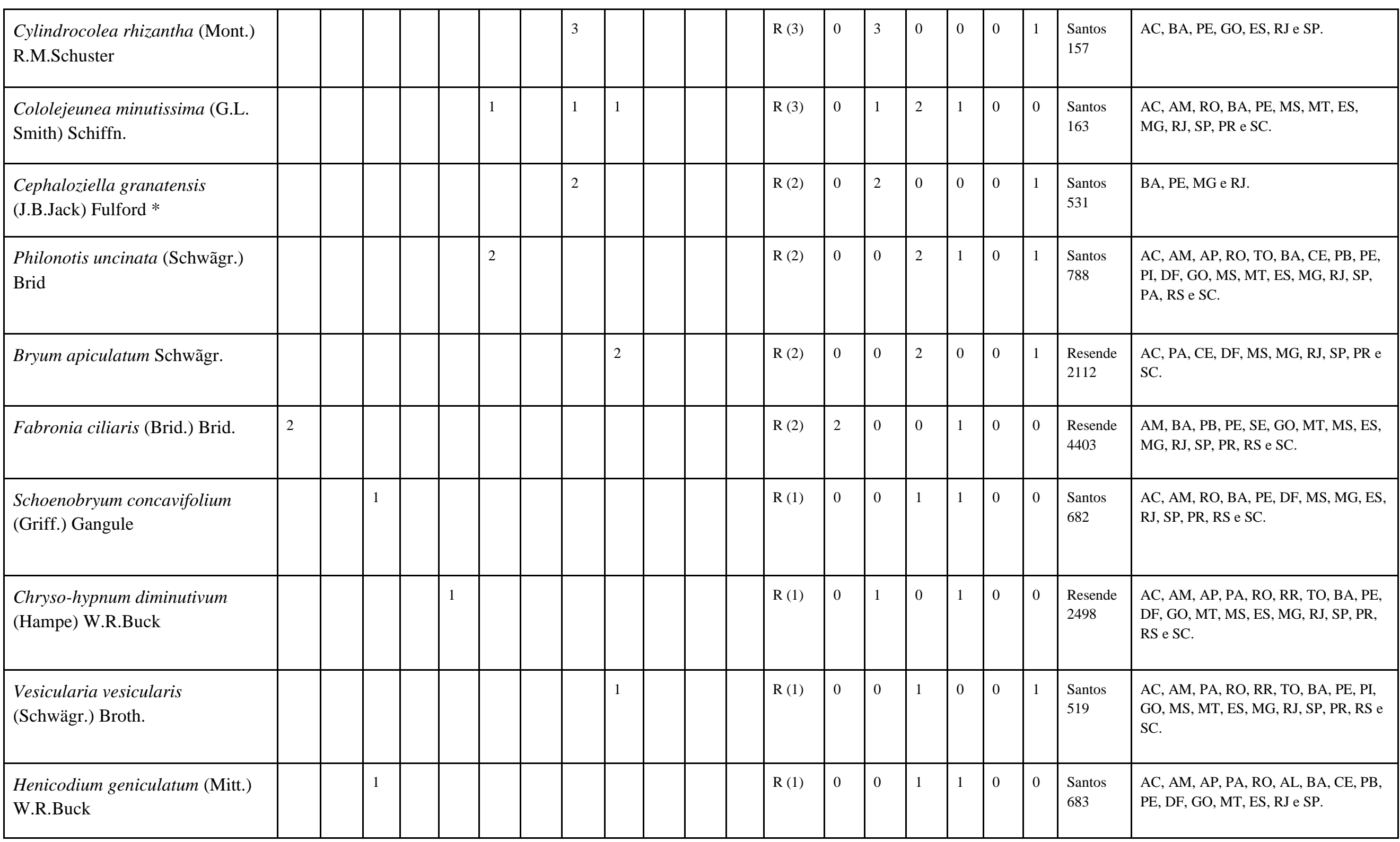




\begin{tabular}{|c|c|c|c|c|c|c|c|c|c|c|c|c|c|c|c|c|c|c|c|c|c|}
\hline Barbula arcuata Griff. & & & & & & & & & & & 1 & & $\mathrm{R}(1)$ & 0 & 1 & 0 & 0 & 0 & 1 & $\begin{array}{l}\text { Santos } \\
316\end{array}$ & AM, GO, MG, RJ e SP. \\
\hline $\begin{array}{l}\text { Entodontopsis leucostega (Brid.) } \\
\text { W.R.Buck \& Ireland }\end{array}$ & & & & & & & & & 1 & & & & $\mathrm{R}(1)$ & 0 & 0 & 1 & 0 & 0 & 1 & $\begin{array}{l}\text { Resende } \\
2061\end{array}$ & $\begin{array}{l}\text { AC, AM, PA, RO, RR, TO, BA, CE, } \\
\text { MA, PB, PE, PI, DF, GO, MS, MT, MG, } \\
\text { RJ e SP. }\end{array}$ \\
\hline Lophocolea platensis C. Massal. * & & & & & & & & & & & 1 & & $\mathrm{R}(1)$ & 0 & 1 & 0 & 1 & 0 & 0 & $\begin{array}{l}\text { Santos } \\
509\end{array}$ & GO, RS, SC e SP. \\
\hline Frullania caulisequa (Nees) Nees & & & & & & & & 1 & & & & & $\mathrm{R}(1)$ & 0 & 1 & 0 & 1 & 0 & 0 & $\begin{array}{l}\text { Santos } \\
155\end{array}$ & $\begin{array}{l}\text { AC, PA, RO, AL, BA, CE, PB, PE, SE, } \\
\text { DF, GO, MT, ES, MG, RJ, SP, RS e SC. }\end{array}$ \\
\hline $\begin{array}{l}\text { Frullania riojaneirensis (Raddi) } \\
\text { Angstr. }\end{array}$ & & & 1 & & & & & & & & & & $\mathrm{R}(1)$ & 0 & 0 & 1 & 1 & 0 & 0 & $\begin{array}{l}\text { Santos } \\
687\end{array}$ & $\begin{array}{l}\text { PA, BA, CE, PE, SE, DF, GO, MS, MT, } \\
\text { ES, MG, RJ, SP, PR, RS e SC. }\end{array}$ \\
\hline Cololejeunea diaphana A. Evans & & & & & & & & 1 & & & & & $\mathrm{R}(1)$ & 0 & 1 & 0 & 1 & 0 & 0 & $\begin{array}{l}\text { Santos } \\
1768\end{array}$ & $\begin{array}{l}\text { AM, PA, PE, GO, MT, ES, RJ, SP, RS e } \\
\text { SC. }\end{array}$ \\
\hline $\begin{array}{l}\text { Symphyogyna aspera Steph. \& } \\
\text { McCormick }\end{array}$ & & & & & & & & 1 & & & & & $\mathrm{R}(1)$ & 0 & 1 & 0 & 0 & 0 & 1 & $\begin{array}{l}\text { Resende } \\
1910\end{array}$ & $\begin{array}{l}\text { BA, CE, PE, SE, DF, GO, MS, MT, ES, } \\
\text { MG, RJ, SP, PR, RS e SC. }\end{array}$ \\
\hline Octoblepharum albidum Hedw. & & & & & 1 & & & & & & & & $\mathrm{R}(1)$ & 0 & 1 & 0 & 0 & 0 & 1 & $\begin{array}{l}\text { Santos } \\
784\end{array}$ & $\begin{array}{l}\text { AC AM, PA, RO, RR, TO, AL, BA, CE, } \\
\text { MA, PB, PE, PI, RN, SE, DF, GO, MT, } \\
\text { ES, MG, RJ, SP, RS e SC. }\end{array}$ \\
\hline TOTAL & 67 & 202 & 4 & 16 & 173 & 14 & 11 & 139 & 28 & 19 & 191 & 27 & 891 & 113 & 705 & 73 & 24 & 9 & 23 & & \\
\hline
\end{tabular}

Fonte: Autores. 
Figura 2. A curva de rarefação considerou os dados florísticos para as quatro veredas.

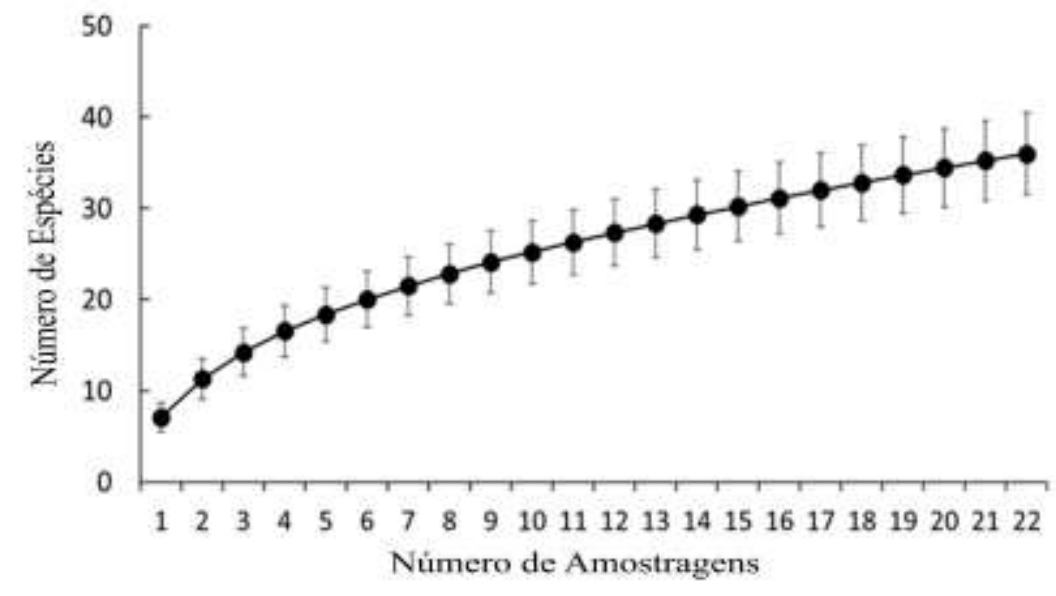

Fonte: Autores.

As espécies encontradas estão amplamente distribuídas no território brasileiro (Tabela 2) quando comparadas com a listagem apresentada na Flora do Brasil (2020), porém, ainda assim, estamos apresentando quatro novas ocorrências para o estado de Goiás (marcada com * na tabela 2) e duas novas ocorrências para Minas Gerais (marcada com ** na tabela 2).

A frequência das espécies pode ser agrupada em dois grupos, as raras: incluindo a maioria das espécies $(n=21)$ classificada como rara (R) e quatro como Pouco Frequentes (P), e as abundantes, incluindo as sete Muito Comuns (M), Assíduo (A) e Frequente (F) com duas espécies cada (Tabela 2). O número de espécies de briófitas em cada uma das classes de frequência (Figura 3), tomou a forma de um "J" invertido como esperado para comunidades naturais (Ricklefs 2010).

Figura 3. Número de espécies de briófitas por classe de frequência em quatro veredas nos estados de Goiás (V1, V2 e V3) e no Triângulo Mineiro (V4).

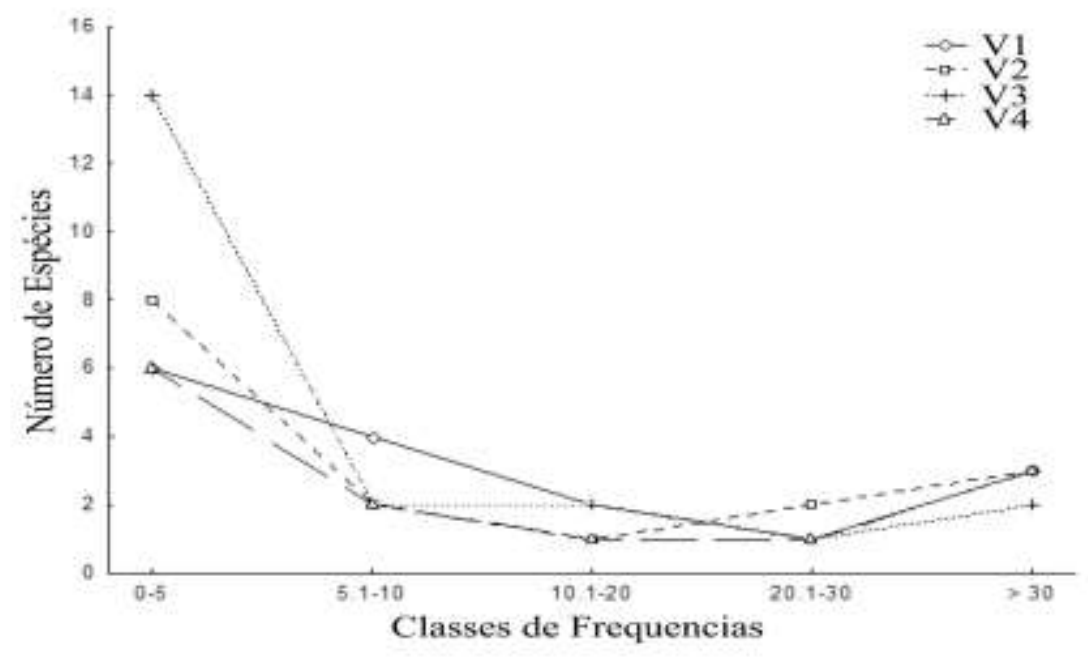

Fonte: Autores.

As sete espécies muito comuns (> 30 ocorrências) foram, em ordem decrescente: Isopterygium tenerifolium (218 amostras), Riccardia chamedryfolia (161 amostras), Lejeunea glaucescens (103 amostras), Ectropothecium leptochaeton (71 amostras), Sphagnum subsecundum (65 amostras), Isopterygium affusum (56 amostras) e Philonotis sphaerocarpa (53 amostras). 
Todas estas espécies são características de ambientes terrestres e úmidos. Isopterygium tenerifolium foi ainda citada por Peralta (2015), Genevro et al. (2006) e Carvalho-Silva et al. (2010) como generalista por colonizar diversos substratos.

A colonização de briófitas concentrou-se em três tipos de substrato com 24 espécies colonizando troncos de árvores vivas (co), 23 espécies colonizando solo (te) e nove colonizando troncos caídos (ep), sendo que destas, a maioria, 23 espécies, colonizou apenas um tipo de substrato e 20 colonizou mais de um, sendo que 13 ocorreram em dois e sete em três substratos (Tabela 2).

A maioria das espécies foi encontrada na base do caule ou bainhas (co) de várias plantas vasculares das veredas. As que se destacaram por abrigar as briófitas foram Cyperaceae, Poaceae, Eriocaulaceae, Melastomataceae, Fabaceae e Asteraceae. Essas famílias estão entre as dez mais ricas em espécies e com maior número de indivíduos nas veredas (Araújo et al. 2002; Resende et al. 2013).

As espécies encontradas colonizando solo já foram mencionadas em outros trabalhos, tais como: Philonotis sphaerocarpa pode ser encontrada em rocha, ambientes secos e em campos rochosos (Valente 2013); Campylopus angustiretis e Ectropothecium leptochaeton ocorrem em solo encharcado e ambientes úmidos (Yano \& Peralta 2005); Isopterygium tenerifolium pode ser encontrada em um ambiente de campo rochoso como cortical, terrestre, rupícola, epíxila ou humícola (Ballejos \& Bastos 2009); Riccardia chamedryfolia cresce em rocha e tronco vivo (Yano \& Peralta 2011a); Lejeunea glaucescens pode ser encontrada com hábito corticícola, terrestre e epixílico (Bastos \& Yano 2009) e Symphyogyna brasiliensis ocorre colonizando solos úmidos, troncos caídos e rochas, em ambientes sombreados (Gradstein \& Costa 2003).

A distribuição das espécies em relação à posição dentro da vereda mostrou que a maioria das espécies foi encontrada no meio (Tabela 2). Isso se deve às condições favoráveis que são disponibilizadas às espécies de briófitas nesta zona. A umidade do solo se destaca no meio e na parte inferior do caminho (Ribeiro \& Walter 2008), sendo o meio o local que apresenta a maior riqueza de espécies vegetais (Resende et al. 2013).

A área do meio é a maior nas veredas estudadas, por isso hospeda mais espécies, além de sofrer menos perturbação que a borda (Guimarães et al. 2002). A borda é a área da vereda que apresenta maior perturbação, sendo comum observar nesta área a presença de drenos, pastagem e pisoteio de gado e espécies invasoras (Oliveira et al. 2009; Resende et al. 2013) Além disso, é o local com solo mais seco, o que não é favorável à reprodução das briófitas.

Dentre as 36 espécies encontradas, apenas quatro (12\%) foram comuns às quatro veredas estudadas: Isopterygium tenerifolium, Riccardia chamedryfolia, Lejeunea glaucescens e Symphyogyna brasiliensis (Tabela 2). Essas espécies também foram as mais abundantes nas áreas de estudo.

Poucas espécies ocorreram nos três ambientes das veredas, com Isopterygium affusum e Telaranea nematodes em V2, Lejeunea glaucescens em V3 e Isopterygium tenerifolium em V4. Pode-se inferir que essas espécies apresentam ampla faixa de tolerância às condições ambientais, com alta plasticidade fenotípica, o que garante maiores chances de adaptação às mudanças ambientais. A plasticidade fenotípica é a habilidade de um organismo de mudar sua fisiologia e ou morfologia em resposta a mudanças nas condições ambientais (Schlichting 1986). Uma estratégia para enfrentar com sucesso o desafio das mudanças ambientais é a geração de uma prole variável gametofítica e esporofítica, capaz de sobreviver em diferentes ambientes. Dessa forma, mesmo que uma parte dos descendentes deixe de existir, a permanência da espécie como um todo estará garantida (Ricklefs 2010).

A Vereda V3 foi a que apresentou maior número de espécies exclusivas com nove espécies, seguida de V1 com quatro, V2 com duas e V4 com uma (Figura 4). 
Figura 4. Dendrograma gerado a partir do índice de similaridade de Jaccard entre as quatro áreas estudadas, em Quirinópolis (V1 e V2), Gouvelândia (V3), Goiás e Monte Alegre de Minas (V4), em Minas Gerais.

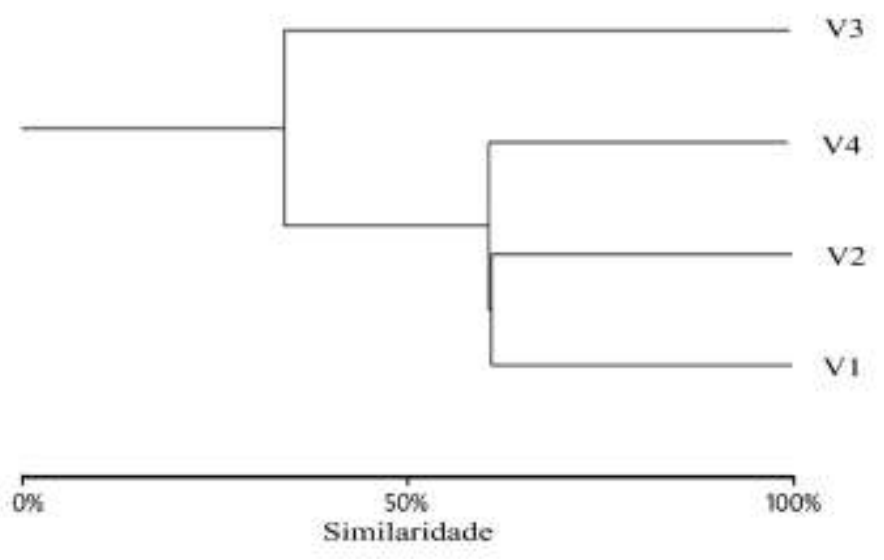

Fonte: Autores.

A análise de agrupamento mostra que as veredas mais semelhantes foram V1, V2 e V4, com $60 \%$ de similaridade. A vereda mais distante ao agrupamento (Figura 4), com a formação de dois grupos, independentes da localização das áreas de veredas. Assim, constatou-se que não houve relação entre a localização geográfica das veredas e a formação dos grupos. Segundo Kent e Coker (1992) valores maiores ou iguais a 50\% indicam alta similaridade. Assim, de acordo com esse conceito, a similaridade analisada entre essas áreas pode ser considerada alta.

A análise do dendrograma mostra que as zonas com alta similaridade são MV4 e MV2 (70\%) e FV4 e BV4 (60\%) (Figura 5), entre as zonas das veredas o Índice de Similaridade foi zero ou baixo entre a maioria das zonas. Portanto, embora tenha havido uma grande semelhança de brioflora entre algumas das veredas estudadas, ao comparar os diferentes ambientes de cada caminho, não há semelhança ou é baixa. Nesse sentido, pode-se inferir que existe uma elevada heterogeneidade ambiental nas veredas, o que reflete a falta de similaridade na distribuição da brioflora nesses ambientes. As diferenças encontradas indicam que as espécies se caracterizam por estarem espacialmente distribuídas em mosaicos e mesmo as comunidades localizadas em áreas próximas são estruturalmente diferenciadas. 
Figura 5. Dendrograma gerado a partir do índice de similaridade de Jaccard entre as doze zonas das veredas estudadas, em Quirinópolis (V1 e V2), Gouvelândia (V3), Goiás e Monte Alegre de Minas (V4), Minas Gerais. Siglas: Bo - borda, Me - meio $\mathrm{e} \mathrm{Fu}$ - fundo.

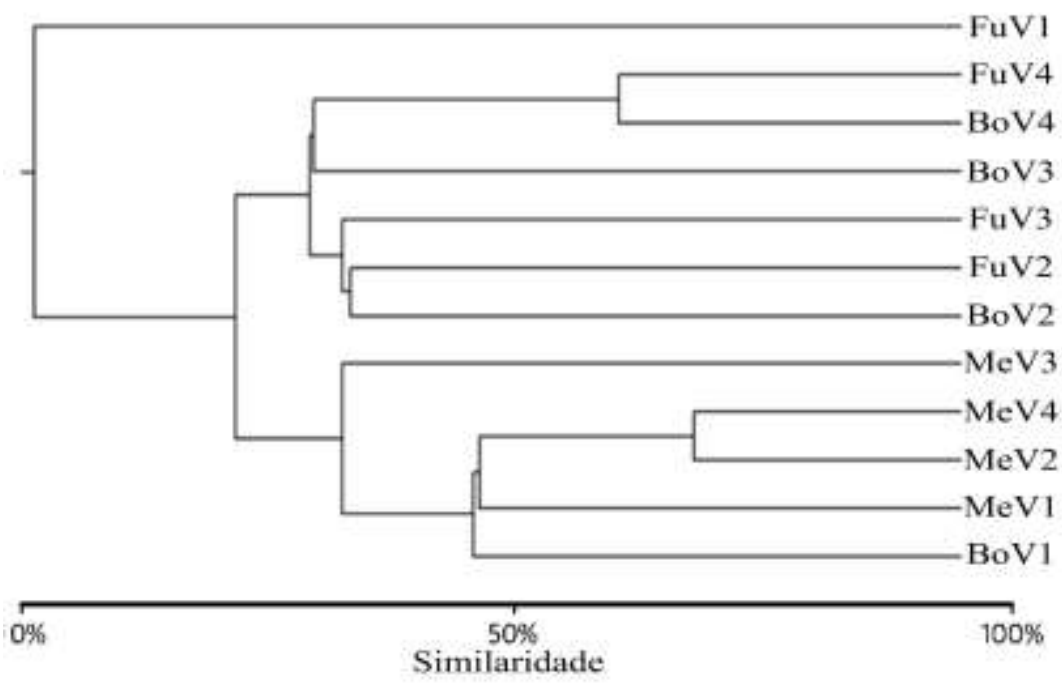

Fonte: Autores.

\section{Considerações Finais}

Os resultados da presente pesquisa mostram que embora tenha ocorrido uma alta similaridade da brioflora entre algumas das veredas estudadas, entre os diferentes ambientes de cada vereda não existe similaridade. Logo, há uma alta heterogeneidade ambiental nas veredas e a inexistência de similaridade entre as zonas das veredas quanto à distribuição da brioflora. As diferenças encontradas indicam que as espécies se caracterizam por se distribuírem espacialmente em mosaicos e mesmo as comunidades situadas em áreas próximas apresentam-se estruturalmente diferenciadas, não existindo relação entre a localização geográfica das veredas e a similaridade da brioflora.

\section{Agradecimentos}

Ao Dr. Wellington Hannibal por ajudar com os dendrogramas e ao Dr. J. Francisco Morales por ajudar com figuras. O segundo autor agradece à Universidade Estadual de Goiás pela bolsa do Programa de Incentivo à Pesquisa e Produção Científica (PROBIP / UEG).

\section{Referências}

Aquino, H. F., Resende, I. L. M., Peralta, D. F., \& Rocha, L. M. (2015). Bryoflora of Gallery Forest in Quirinópolis, Goias State, Brazil. Hoehnea, 42, 419-424. Araújo, G. M., Barbosa, A. A., Arantes, A. A., \&, Amaral, A. F. (2002). Composição florística de veredas no Município de Uberlândia, MG. Revista Brasileira de Botânica, 25, 475-493.

Ballejos, J., \& Bastos, C. J. (2009). Musgos Pleurocárpicos do Parque Estadual das Sete Passagens, Miguel Calmon, Bahia, Brasil. Hoehnea, 36, 479-495.

Bastos, C. J. P., \& Yano, O. (2009). O gênero Lejeunea (Lejeuneaceae) no Estado da Bahia, Brasil. Hoehnea, 36, $259-268$.

Bastos, L. A., \& Ferreira, I. M. (2010). Composições Fitofisionômicas do Bioma Cerrado: estudo sobre o subsistema de Vereda. Espaço em Revista, 12, 97-108. Buck, W. R. (2003). Guide to the plants of Central French Guiana: Mosses. Memoirs of The New York Botanical Garden, 76, 1-167.

Buck, W. R., \& Goffinet, B. (2000). Morphology and classification of mosses. p.p. 71-123. In: Shaw, A. J., \& Goffinet, B. (eds.). Bryophyte Biology. Cambridge University Press, England.

Carmo, D. M. do., \& Peralta, D. F. (2016). Survey of bryophytes in Serra da Canastra National Park, Minas Gerais, Brazil. Acta Botanica Brasilica, 30(2), 254265. 
Carvalho, M. A. S., Santos, X. S., \& Guilherme, F. A. G. (2014). Edge Effects on Epiphytic Moss (Bryophyta) Communities in a Savanna Area in Central Brazil. Brazilian Journal of Ecology, 13, 17-28.

Carvalho-Silva, M., Soares, A. E. R., Câmara, P. E. A. S., \& Dias-Neto, R. G. (2010). Levantamento de musgos (Bryophyta) do Jardim Botânico de Brasília, Distrito Federal, Brasil. Heringeriana, 4, 11-27.

Colwell, R. K. (2013). EstimateS. Version 9.1.0: Statistical estimation of species richness and shared species from samples (Software and User's Guide). Freeware for Windows and Mac OS.

Crandall-Stotler, B., Stotler, R. E., \& Long-Long, D. G. (2009). Phylogeny and classification of the Marchantiophyta. Edinburgh Journal of Botany, 66, 155198.

Crandall-Stotler, B., \& Stotler, R. (2000). Morphology and classification of the Marchantiophyta. p. 21-70. In: Shaw, A.J. \& Goffinet, B. (eds.). Bryophyte Biology. Cambridge University Press, England.

Filgueiras, T. S., Nogueira, P. E., Brocado, A. L., \& Guala II, G. F. (1994). Caminhamento: um método expedito para levantamentos florísticos qualitativos. Caderno de Geociências, 12, 39-43.

Flora do Brasil 2020. (2020). Lista de Espécies da Flora do Brasil. <http://floradobrasil.jbrj.gov.br/>

Frahm, J. P. (2003). Manual of tropical Bryology. Tropical Bryology, 23, 1-196.

Genevro, J. A., Athayde-Filho, F. P., \& Peralta, D. F. (2006). Briófitas de mata de galeria no Parque Municipal Mário Viana, Nova Xavantina, Mato Grosso, Brasil. Boletim do Instituto de Botânica, 18, 149-157.

Goffinet, B., Buck, W. R., \& Shaw, A. J. (2009). Morphology, anatomy and classification of the Bryophyta.p.p. 56-138. In: Goffinet, B., \& Shaw, A. J. Bryophyte Biology. Second Edition. Cambridge University Press.

Gradstein, S. R. (1994). Lejeuneaceae; Ptychantheae, Brachiolejeuneae. Flora Neotropica, Monograph, 62, 1-216.

Gradstein, S. R., Churchill, S. P., \& Salazar-Allen, N. (2001). Guide to the Bryophytes of Tropical America. Memoirs of The New York Botanical Garden, 86, $1-577$.

Gradstein, S. R., \& Costa, D. P. (2003). The Hepaticae and Anthocerotae of Brazil. Memoirs of The New York Botanical Garden, 87, 1-318.

Guimarães, A. J. M., Araújo, G. M., \& Corrêa, G. F. (2002). Estrutura fitossociológica em área natural e antropizada de uma vereda em Uberlândia, MG. Acta Botanica Brasilica, 16, 317-329.

Hammer, O., Harper, D. A. T., \& Ryan, P. D. (2001). Past: paleontological statistics software package for education and data analysis. Palaeontologia Electronica, 4, 1-9.

Kent, M., \& Coker, P. (1992). Vegetation Description and Analysis. Belhaven Press, London.

Luizi-Ponzo, A. P., Siviero, T. S., Amorim, E. T., Henriques, D. K., Rocha, L. M., Gomes, H. C. S., Paiva, L. A., Rodrigues, R. S., Silva, I. C., Silva, A. G. D., Ribeiro, G. C., Gomes, C. Q., \& Campeão, A. S (2013). Briófitas do Parque Estadual do Ibitipoca no Herbário Prof. Leopoldo Kriegeer. In: Forzza, R. C., Neto, L. M., Salimena, F. R. G., \& Zappi, D. (Orgs.). Flora do Parque Estadual do Ibitipoca e seu entorno. 1ed. Juiz de Fora: Editora UFJF, v. 4, p. 95-122.

Mueller-Dombois, D., \& Ellenberg, H. (1974). Aims and methods of vegetation ecology. John Wiley and Sons, New York.

Oliveira, G. C., Araújo, G. M., \& Barbosa, A. A. A. (2009). Florística e zonação de espécies vegetais em veredas no Triângulo Mineiro, Brasil. Rodriguésia, 60, $1077-1085$

Peralta, D. F. (2015). Briófitas. In: Lista de Espécies da Flora do Brasil. Jardim Botânico do Rio de Janeiro. <http://reflora.jbri.gov.br/jabot/floradobrasil/FB128472>.

Pursell, R. A. (2007). Fissidentaceae. Flora Neotropica, Monograph, 101, 1-278.

Reese, W. D. (1993). Calymperaceae. Flora Neotropica, Monograph, 58, 1-102.

Resende, I. L. M., Chaves, L. J., \& Rizzo, J. A. (2013). Floristic and phytosociological analysis of palm swamps in the central part of the Brazilian savanna. Acta Botanica Brasilica, 27, 205-225.

Ribeiro, J. F., \& Walter, B. M. T. (2008). As principais fitofisionomias do Bioma Cerrado. p.p. 151-212. In: Sano, S. M., Almeida, S. P., \& Ribeiro, J. F. (Eds.). Cerrado: Ecologia e Flora. Embrapa, Brasília. v. 1.

Ricklefs, R. (2010). A Economia da Natureza. 6 ed. Editora Guanabara Koogan, Rio de Janeiro.

Robbins, R. G. (1952). Bryophyte Ecology of a dune area in New Zealand. Vegetation. Acta Geobotanica, 4, 1-31.

Schlichting, C. D. (1986). The evolution of phenotypic plasticity in plants. Annual Review of Ecological and Systematics, 17, 667-693.

Semarh (Secretaria De Estado Do Meio Ambiente E Dos Recursos Hídricos). (2005). Texto final das Conferências Estaduais de Meio Ambiente. Tema IV: Gestão Territorial e Política Florestal. SEMARH, Pirenópolis.

Sharp, A. J., Crum, H., \& Eckel, P. M. (1994). The moss flora of Mexico. Memoirs of The New York Botanical Garden, 69, 1-1113. 
Shepherd, G. J. (2003). Avaliação do estado do conhecimento da diversidade biológica do Brasil: Plantas Terrestres. Departamento de Botânica, Instituto de Biologia, Universidade Estadual de Campinas, p.1-59.

Silva, M. P. P., \& Pôrto, K. C. (2007). Composição e riqueza de briófitas epíxilas em fragmentos florestais da Estação Ecológica de Murici, Alagoas. Revista Brasileira de Biociências, 5, 243-245.

Silva, T. A., \& Maillard, P. (2011). Delimitação, caracterização e tipologia das veredas do Parque Estadual Veredas do Peruaçu. Geografias artigos científicos, 7, 24-39.

Sousa, M. A. R. V. L, Gomes-Klein, V. L., \& Yano, O. (2010). Musgos (Bryophyta) do Parque Estadual da Serra dos Pireneus, Goiás, Brasil. Revista Biologia Neotropical, 7, 7-26.

Sousa, R. V., \& Câmara, P. E. A. S. (2015). Survey of Bryophytes of a gallery forest in the National Park of Serra do Cipó, Minas Gerais, Brazil. Acta Botanica Brasilica, 29, 24-29.

Stoller, R. E., \& Crandall-Stoler, B. (2005) A revised classification of the Anthocerotophyta and a checklist of the horworts of North America, North of Mexico. The Bryologist, 108, 16-26.

Valente, E. B., Pôrto, K. C., \& Batos, C. J. P. (2013). Species richness and distribution of bryophytes within different phyto physiognomies in the Chapada Diamantina region of Brazil. Acta Botanica Brasilica, 27, 294-310.

Vanderpoorten, A., \& Goffinet, B. (2009). Introduction of Bryophytes. Cambridge University Press, Cambridge.

Venn, J. (1984). Symbolic Logic. (2a ed.) Macmillan Press, London.

Vital, D. M. (1980). Erpodiaceae (Musci) do Brasil. Dissertação de Mestrado, Universidade Estadual de Campinas, Campinas.

Yano, O. (1975). Leucobryaceae (Musci) do Estado de São Paulo. Dissertação. Escola Paulista de Medicina São Paulo, São Paulo.

Yano, O., \& Carvalho, A.B. (1995). Briófitas da Serra da Piedade, Minas Gerais, Brasil. p.15-25. In: Anais do $9^{\circ}$ Congresso da Sociedade Botânica de São Paulo.

Yano, O., \& Peralta, D. F. (2005). Briófitas de mata paludosa, município de Zacarias, noroeste do Estado de São Paulo. Acta Botanica Brasilica, 19 , 963-977.

Yano, O., \& Peralta, D. F. (2009). Flora de Grão-Mogol, Minas Gerais. Briófitas (Bryophyta e Marchantiophyta). Boletim de Botânica da Universidade de São Paulo, 27, 1-26.

Yano, O., \& Peralta, D. F. (2011a). Bryophytes from Serra de São José, Tiradentes, Minas Gerais, Brasil. Boletim de Botânica da Universidade de São Paulo, 21, 141-172.

Yano, O., \& Peralta, D. F. (2011b). Flora da Serra do Cipó, Minas Gerais: Briófitas (Anthocerotophyta, Bryophyta e Marchantiophyta). Boletim de Botânica da Universidade São Paulo, 29, 135-299. 